\title{
Complex Morphostructural and in vitro Biological Investigation of Nanocomposite Hydrogels Tailored with Magnetic Nanoparticles
}

\author{
EUGENIA TANASĂ ${ }^{1}$, IONUT-CRISTIAN RADU ${ }^{2}$, BIANCA GALATEANU ${ }^{3}$, ARIANA HUDITA ${ }^{3}$, \\ ECATERINA ANDRONESCU ${ }^{1}$, CATALIN ZAHARIA ${ }^{2 *}$ \\ ${ }^{1}$ Politehnica University of Bucharest, Faculty of Applied Chemistry and Materials Science, 1-7 Polizu Str., 060042, \\ Bucharest, Romania \\ ${ }^{2}$ Politehnica University of Bucharest, Advanced Polymer Materials Group, 1-7 Gh. Polizu Str., 011061, Bucharest, \\ Romania \\ ${ }^{3}$ University of Bucharest, Department of Biochemistry and Molecular Biology, 91-95 Splaiul Independentei, 050095, \\ Bucharest, Romania
}

The morphology and the microstructure of the nanocomposite hydrogels, prepared by free-radical polymerization of acrylamide monomer and double bond functionalized magnetite nanoparticles, were analysed by scanning electron microscopy. Nanostructural characteristic and crystalline structure were studied by high resolution transmission electron microscopy and selected area electron diffraction. Elemental composition was analysed by energy dispersive $X$-ray spectroscopy. In vitro biocompatibility evaluation was performedon specific cell lines.

Keywords: magnetic nanoparticles, polyacrylamide, SEM, TEM, biocompatibility

During the last decade,nanoscience represented one of the most researched fields and promoted a new concept, nanotechnology. Thus, the nanotechnology concept aims that the work of engineers, chemists or biologists to meet at cellular and molecular level. In this regard, the use of nanoparticles offered new prospects due to their unique size and chemical reactivity [1-3]. Nanoparticles have many applications in medicine, like filler for the bone tissue engineering scaffolds [4], scaffolds for dentistry [5], coating layers on metallic surfaces [6] or solutions for treatment of chondral injuries [7]. First, they may exhibit controllable size in the range of few nanometres up to $100 \mathrm{~nm}$, being comparable with biological parts of cells (proteins, genes, etc.). Considering the biological parts of cells to be biological entity of interest, the nanoparticles can get close interactions with them. Furthermore, magnetic nanoparticles based on metal oxides such as iron, nickel or cobalt can be manipulated at a distance by an external magnetic field [8,9]. These unique characteristics and needs for non-invasive therapy with high effective management ensured a fast progress of such complex field. Thus, the permanent development of imaging techniques, such as electronic microscopy,fulfils the already success by improving the contrast and highlighting the key role of nanoparticles in connection with tissues beyond all expectation [10-14].

Iron oxide nanoparticles, such as magnetite, represent by far the most known and used magnetic nanoparticles in the biomedical field. They are non-toxic and non-immunogenic nanoparticles, able to comprise real uses in life science for various nanostructured materials $[15,16]$. Furthermore, magnetic nanoparticles are able to offer a resonantly time response for variation of magnetic field gradient. In biomedical uses, such as tissue engineering, magnetic nanoparticles (e.g. magnetite) are preferred because they exhibit paramagnetic behaviour at low temperature, present stability in water at $\mathrm{pH} 7$ and biological environments [17-19].

In this paper we address a complex morphostructural and biological investigation of the previously developed nanocomposite hydrogels. The complex investigation focuses a micro- and nanostructural characterization by modern techniques (SEM, TEM, SAED, BF-TEM, HRTEM). The morphological characterization is followed by a biological assay aiming to support the very promising use in the tissue engineering field.

\section{Experimental part}

Materials and methods

All the reagents were purchased from Sigma-Aldrich. Magnetic nanoparticles were synthesized starting from iron chloride iron (III) chloride $\left(\mathrm{FeCl}_{3}, 97 \%\right)$, ferrous sulfateheptahydrate $\left(\mathrm{FeSO}_{4} \cdot 7 \mathrm{H}_{2} \mathrm{O}\right)$ and ammonium hydroxide solution $\left(\mathrm{NH}_{4} \mathrm{OH}\right)$. Acrylamide, potassium persulfate (KPS) and3-trimethoxysilyl propyl methacrylate were used for the synthesis of the hydrogels.

Magnetic nanocomposites were obtained from double bond modified magnetite nanoparticles (MMNPs) and acrylamide by redox polymerization reaction. The synthesis of modified magnetic nanoparticles was described elsewhere [20]. The preparation of the nanocomposites was performed by free-radical polymerization of acrylamide and

*email: zaharia.catalin@gmail.com, Phone: +40 214022715 
MMNPs in aqueous solution [20]. Briefly, 15 wt. \% acrylamide aqueous solution was prepared, and MMNPs were added after vigorous sonication in distilled water. Potassium persulfate (KPS) was used as initiator for radical polymerization of acrylamide and MMNPs at $60^{\circ} \mathrm{C}$. The as prepared nanocomposites were purified by washing with distilled water and dried overnight at $40{ }^{\circ} \mathrm{C}$. Various ratios between acrylamide and MMNPs were used for the preparation of nanocomposite hydrogels: 90-10, 80-20, 70-30 and 60-40 w/w (PAA-MMNPs).

Advanced morphological investigation of the nanocomposites by scanning electron microscopy (SEM) and transmission electron microscopy (TEM)

The microstructure of the samples was examined by Scanning Electron Microscopy (SEM) using a Quanta Inspect F50, with a field emission gun (FEG) having $1.2 \mathrm{~nm}$ resolution and an Energy Dispersive X-ray Spectrometer (EDXS), with $133 \mathrm{eV}$ resolution at $\mathrm{MnK} \alpha$. Morphology, geometrical evaluationof nanostructural characteristics and the crystalline structure of the materials were investigated by High Resolution Transmission Electron Microscopy (HRTEM) and selected area electron diffraction (SAED) using a TECNAI F30 $\mathrm{G}^{2}$ S-TWIN microscope operated at 300 kV with Energy Dispersive X-ray Analysis (EDAX) facility.

In vitro biocompatibility evaluation of the nanocomposite hydrogels on specific cell lines

The biocompatibility of PAA-MMNPs nanocomposites was evaluated on mouse preosteoblast cells (3T3-E1 ATCC). Cell viability was investigated by quantitative spectrophotometric studies (MTT test). Cytotoxic potential on 3T3-E1 cells was also investigated at $24 \mathrm{~h}$ and 5 days after cell seeding on nanocomposite materials.

\section{Results and discussions}

SEM analysisof the PAA-MMNPs nanocomposites

SEM was used as an useful tool for morphostructural characterization of the PAA-MMNPs hydrogels in cross section after cooling in liquid nitrogen. Lyophilized specimens of the hydrogels were also analysed by SEM after a freezedrying process. The microstructure of the sample PAA-MMNPs: 80-20is shown in figure 1A and 1B.White contrast areas correspond to high atomic number elements, whereas dark areas are specific to materials with low atomic number elements within the composition. It is clear that the submicronic white zones are specific for magnetite nanoparticles, uniformly distributed in the polyacrylamide matrix (darker areas). This reveals a composite material with two microstructural constituents(figure 1A, backscattered electron image-BSEI, x1.000). The secondary electron image (SEI) in figure $1 \mathrm{~B}$, at $200.000 \mathrm{x}$ magnification, is a detail from the magnetite agglomerations embedded in the PAA matrix. Resolution of such details allow the observation of nanostructural characteristics of the sample. Some spherical particles (magnetite nanoparticles) are seen in these agglomerations, but also a structured matrix in which ribs are embedded in the polymeric phase.

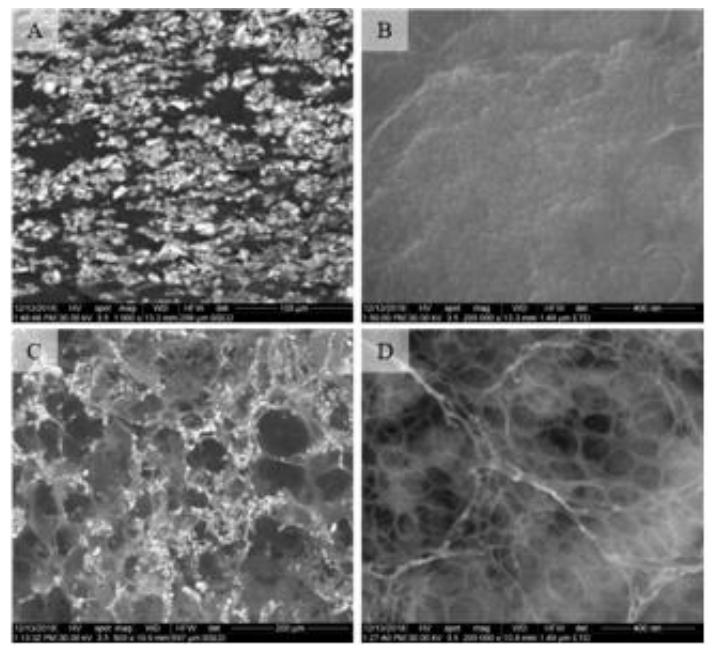

Fig. 1. SEM images: cross section of PAA-MMNPs: 80-20 sample: A) BSEI, $\mathrm{x} 1.000$; B) SEI, x200.000); C), D) cross section of PAA-MMNPs: 80-20 lyophilized sample: C) BSEI, x500; D) SEI, x200.000

Microstructural characteristics of the PAA-MMNPs: 80-20 lyophilised hydrogel are highlighted in figure 1C and 1D.Figure1C (BSEI, x500) reveals the porous structure of the lyophilised sample. The aspect seems uniform with interconnected closed pores of 50-100 $\mu \mathrm{m}$. The microstructural detail in figure 1Cshows the presence of the two constituents: magnetite in white phase contrast and the polymeric PAA matrix which forms the walls of the pores.

Details of the lyophilised nanocomposite microstructure for PAA-MMNPs (80-20) are offered by figure 1D. At higher magnifications (SEI, x200.000), the presence of a spatial nanostructure could be seen as a woven mesh. This mesh is composed of fibrils with bump deformations which could be attributed to the nano-magnetite particles embedded 
in the fibrils. Behind this thin layer of polymer (the mesh), embedded in the wall, it can be observed the magnetite agglomerations with submicronic up to micron dimensions.

Figure 2 shows some edifying results for the microstructure of the PAA-MMNPs: 70-30 sample. At a magnification of 1.000x, the BSE image from figure 2A, of a microarea in this sample, reveals a microstructure similar to the other samplepreviously described in the paper. The microstructure is characteristic to a composite, in the PAA polymer matrix being distributed relatively evenly agglomerations of magnetite nanoparticles. Also, the micro- and nanostructural details revealed by the SE image at higher magnification (figure 2B, 100.000x), highlight the nanostructure of the two structural constituents of the nanocomposite. Similar to the previous sample, the polymeric matrix is made of a mesh build like a network of nanofibers, a network that presents magnetite nanoparticles embedded in them. It can be observed how these polymeric ribs are present also inside the magnetite agglomerations, as shown in figure $2 \mathrm{C}$, at $400.000 \mathrm{x}$ magnification. This image highlights the spherical shape of the nanoparticles, being measured three of the nanoparticles, having diameters of $9.5 \mathrm{~nm}, 11.7 \mathrm{~nm}$ and $13.1 \mathrm{~nm}$.
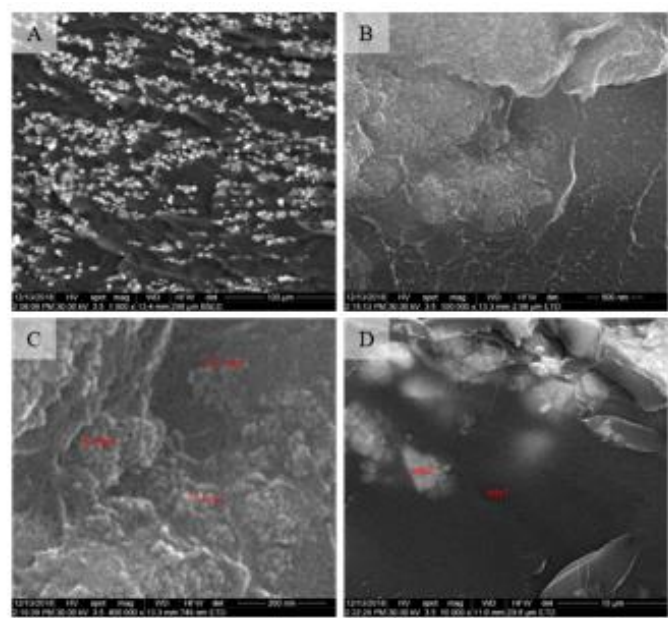

E
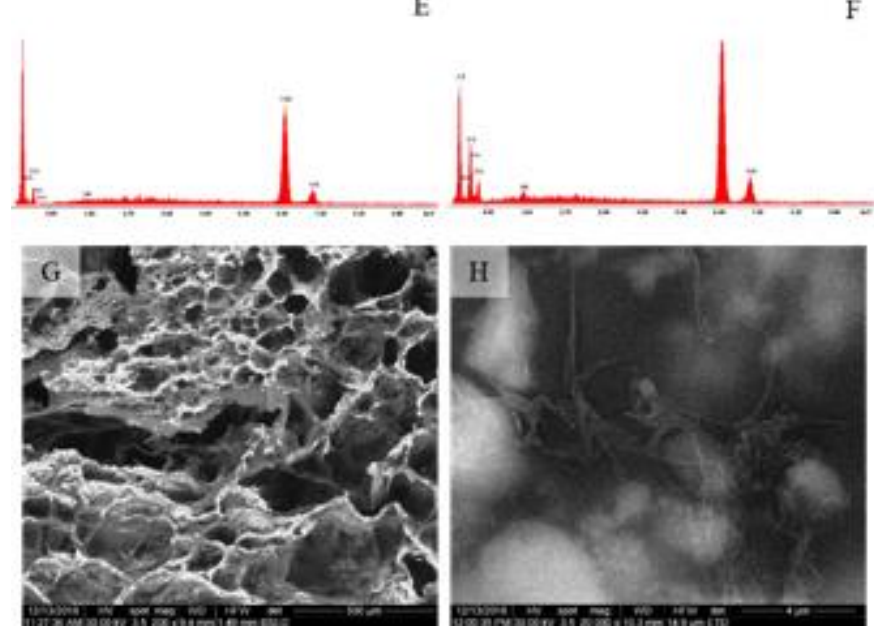

Fig. 2. SEM images: cross section of PAA-MMNPs: 70-30 sample: A) BSEI, x1.000; B) SEI, x100.000; C) SEI, x400.000;

D) SEI, x10.000; E, F) EDX spectra associated to the highlighted areas in image D; Cross section oflyophilized PAA-MMNPs: 70-30 sample: G) BSEI, x200; H) SEI, x20.000

For this sample, the Energy Dispersive X-ray (EDX) analysis was employed in order to demonstrate the composition of the two constituents. Thus, the spectrum in figure $2 \mathrm{E}$ was acquired on the nanoarea marked in figure 2D with the text "edx1", so it gives the elemental composition of the matrix and the spectrum in figure $2 \mathrm{~F}$ was acquired on the nanoarea marked in figure 2D with the text "edx2", so it gives the elemental composition of the magnetite agglomeration. These spectra show the presence of the same elements in the two nanoareas: carbon, oxygen, silicon and iron, with the observation that the relative intensities of the element are different in the two areas. Thus, in the nanoarea labelled "edx1", the ratio of the $\mathrm{C} / \mathrm{Fe}$ intensities is bigger than 1 , so in the corresponding nanoarea the major element present is carbon, a component element of the polymeric matrix. In the nanoarea labelled "edx2", the radio of the C/Fe intensities is smaller than 1 , so in the corresponding nanoarea the major element present is iron, a component element of the magnetite. The fact that in this area there also is the silicon element, with a bigger percentage than in the "edx 1 " nanoarea, attests that the present magnetite is functionalized.

The BSE image of the lyophilized PAA-MMNPs:70-30 sample (figure 2G) confirms the biphasic composition of the material: a polymeric matrix that builds the pore walls and agglomerations of magnetite that seem to be embedded in the polymeric matrix. Similar to the previously lyophilized sample, at higher magnification $(20.000 \mathrm{x}$, figure $2 \mathrm{H})$, there are mesh microstructures build out of polymeric nanofibers that have $\mathrm{Fe}_{3} \mathrm{O}_{4}$ nanoparticles embedded.

The micro- and nanostructural characteristics of the PAA-MMNPs: 60-40are shown in figure 3B (BSEI, x1000), revealing the two components of the hydrogel. As previously described for the other compositions, magnetic nanoparticle agglomerations are embedded and uniformly distributed in the polymeric matrix. Magnetic clusters are composed of magnetite particles of 10-12 $\mu$ m. The polymeric nanofibrils have the same nanostructure that contain $\mathrm{Fe}_{3} \mathrm{O}_{4}$ nanoparticles (figure 3B, SEI, x100.000).Here, the mesh nanostructure is denser (number of fibrils/unit area) as compared to the structure of PAA-MMNPs 80-20 and 70-30.

The characteristics of the lyophilised PAA-MMNPs (60-40) hydrogel are shown in figure 3C and 3D. One may notice in figure $3 \mathrm{C}$ (BSEI, x1.000) the porous microstructure of the sample. Closed and open pores with interconnected structure are observed. There is also shown the biphasic composition of the composite hydrogel. There is a dark contrast phase specific for light elements in the composition, namely the polymeric matrix. It can also be seen a bright contrast phase characteristic to heavy elements such as iron from magnetite, in our case. The SE image (figure $3 \mathrm{D}$, $\mathrm{x} 50.000$ ) is 
a detail of a nanozone of a pore wall. This image certifies the existence of a fibrillary spatial nanostructure (mesh-like), same as the others compositions.
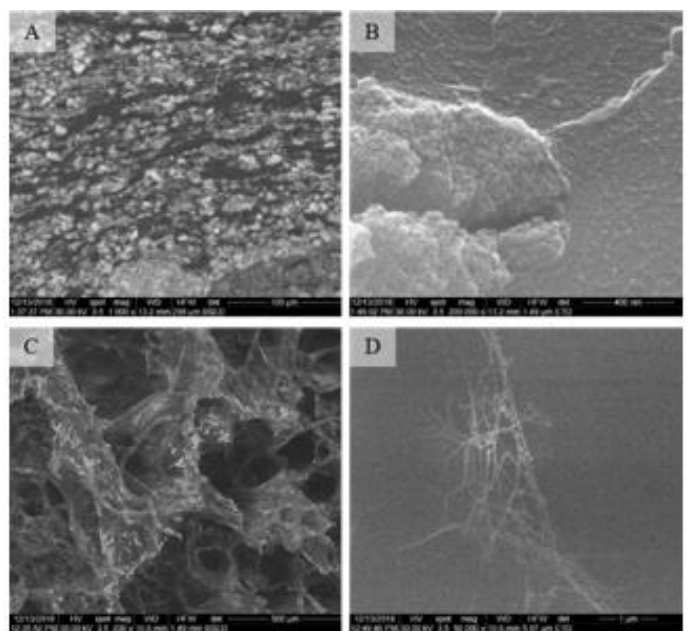

Fig. 3. SEM images - Cross section of PAA-MMNPs: 60-40 sample: A) BSEI, x1.000; B) SEI, x200.000; Cross section of PAA- MMNPs: 60-40 lyophilized sample: C) BSEI, x200; D) SEI, $\mathrm{x} 50.000$

\section{TEM analysisof functionalized magnetic nanoparticles and PAA-MMNPs nanocomposites}

The microstructure and especially the internal nanostructure of the nanocomposite hydrogels were investigated by TEM analysis, which offers information on the localization and the distribution of the magnetic nanoparticles within the polyacrylamide matrix. SAED and HRTEM were employed to obtain qualitative and quantitative information about the phase crystallinity in the nanocomposites.

Magnetic nanoparticles were functionalized with double bonds in this research study as described in the experimental section. To prove this modification, TEM images of double bond modified nanoparticles are presented here (figure 4).
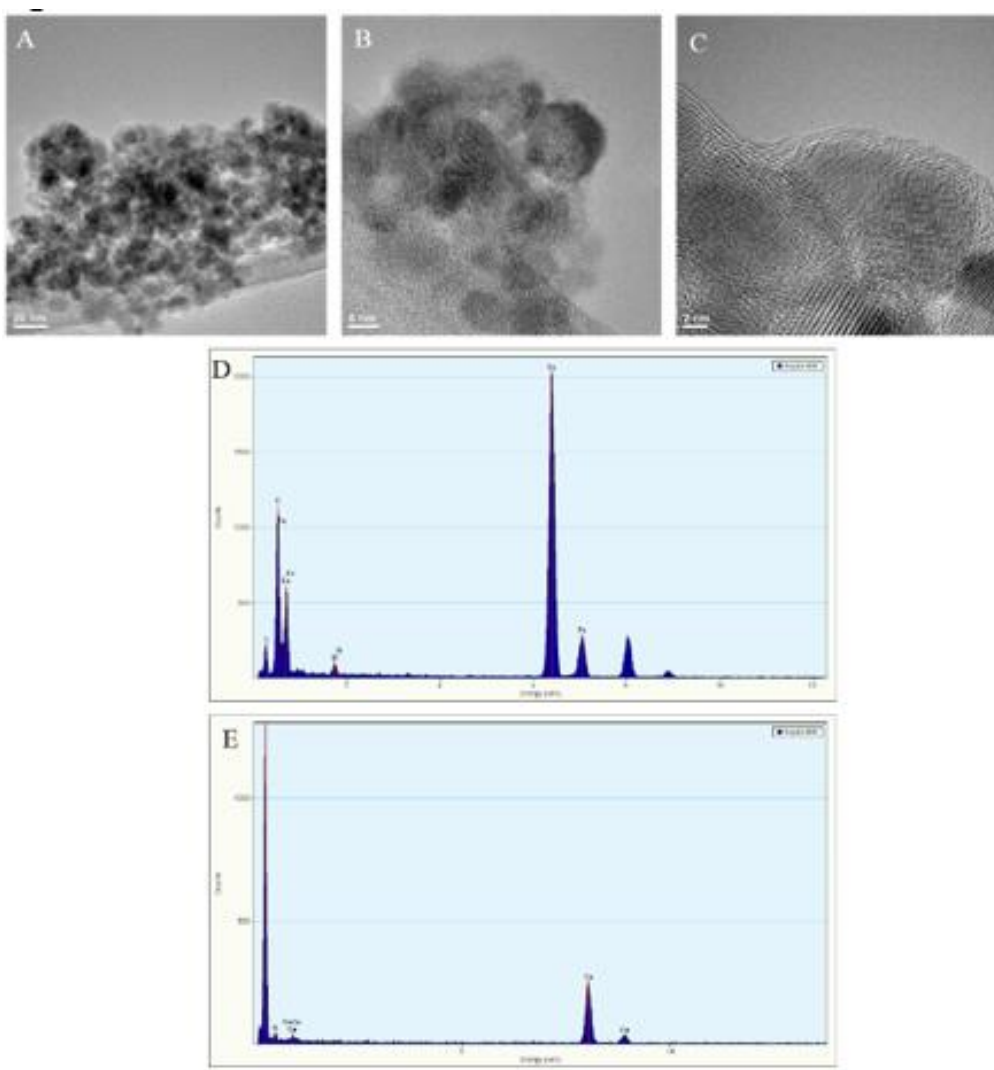

Fig. 4. A), B) and C) TEM

images of double bond modified magnetite (MMNPs); C) EDAX spectrum acquired on the nanoparticles agglomeration from image A; D) EDX spectrum acquired on the carbon film, corresponding to the upper part from image $\mathrm{A}$

The bright field TEM (BF-TEM) image in figure 4A reveals some agglomerations of functionalized magnetic nanoparticles. These MMNPs have almost spherical shape with diameters around 5-9 nm clearly visible in figure 4B and $4 \mathrm{C}$ (HRTEM). Nanoparticles are all part of the same nanocrystalline phase $\left(\mathrm{Fe}_{3} \mathrm{O}_{4}\right.$ compound with face-centred cubic crystalline structure). EDAX spectra were collected on MMNPs agglomerations (figure4D) and on polyacrylamide with amorphous carbon region (figure4E). Fe and $\mathrm{O}$ are major elements in EDAX spectrum shown in figure 4D, but there also some minor elements like carbon, copper and silicon. This proves that magnetite is the main compound in the agglomerations. Silicon ( $\mathrm{Si}$ ) is present only in the thin layer that covers the magnetic nanoparticles. As a conclusion, 
after the functionalization process of the magnetic nanoparticles, a thin organic layer (with double bonds) is attached around the MNPs. The functionalization process does not modify the crystalline structure of the nanoparticles.
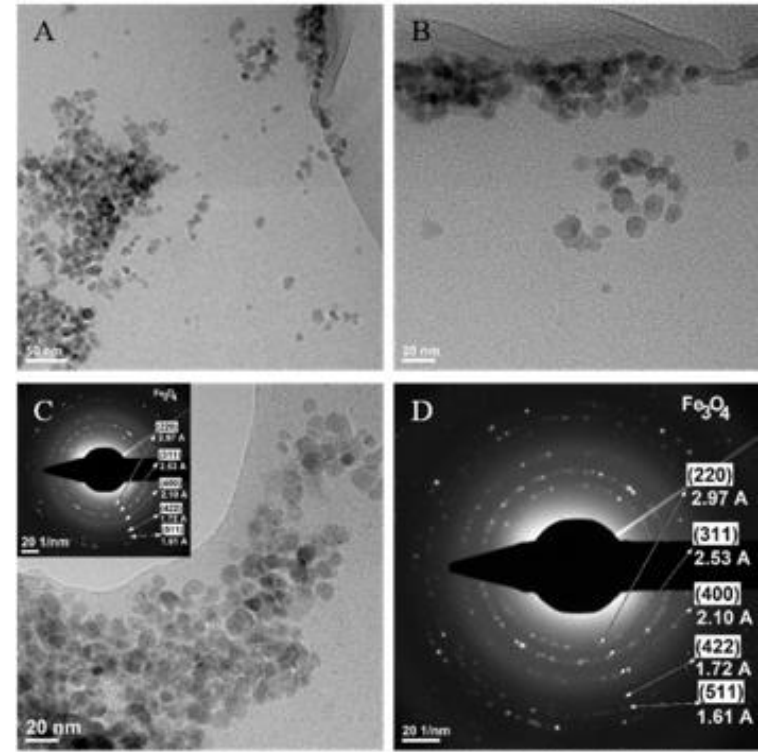

Fig. 5. TEM images of nanocomposite hydrogel PAA-MMNPs (60-40): A), B) and C) BF-TEM images; D) SAED image associated to the nanoarea in image D; E), F) HR-TEM images
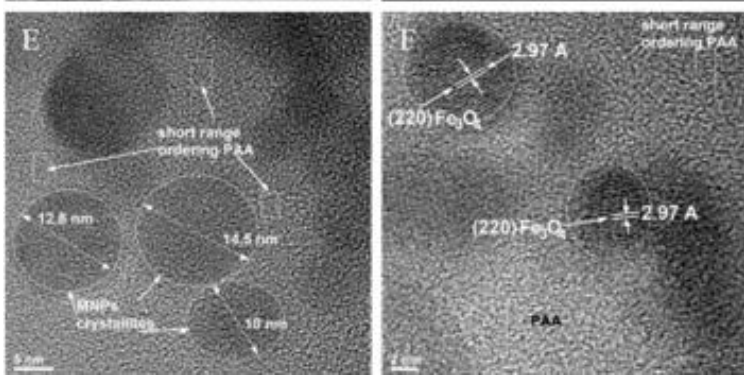

Figure 5 shows TEM images of nanocomposite hydrogel PAA-MMNPs (60-40). Dark contrast zones are functionalized magnetite particles within the PAA matrix.

BF-TEM images (figure5B and 5C) show MMNPs in agglomerations, but also as individual isolated nanoparticles. Even in the agglomerations there is a tendency of the nanoparticles to separate. The separation layer may also contain the organic film that surrounds the MMNPs as a result of functionalization. SAED image (figure 5D) confirms the nanocrystalline nature of the particles.Similar conclusions could be drawn from HRTEM images (figure 5E and 5F). They put into evidence the crystalline magnetic nanoparticles of spherical shape, with diameters between 10 and $15 \mathrm{~nm}$. There are also some nanoregions where PAA has a certain degree of order. The crystalline planes (220), with $2.97 \AA$ interplanar distance are highlighted in figure 5F, confirming that nanoparticles belong to $\mathrm{Fe}_{3} \mathrm{O}_{4}$ phase.

\section{In vitro biocompatibility results}

MTT results are shown in figure 6. The graphs aredrawnin GraphPrism software. MTT results on 3T3-E1 cells exhibited a different profile for cell viability as a function of materials composition. The cell viability increases with the decrease of the PAA amount in the hydrogel composition, and 3T3-E1 have proliferated only in the contact with compositions PAA-MMNPs:60-40 and 50-50.

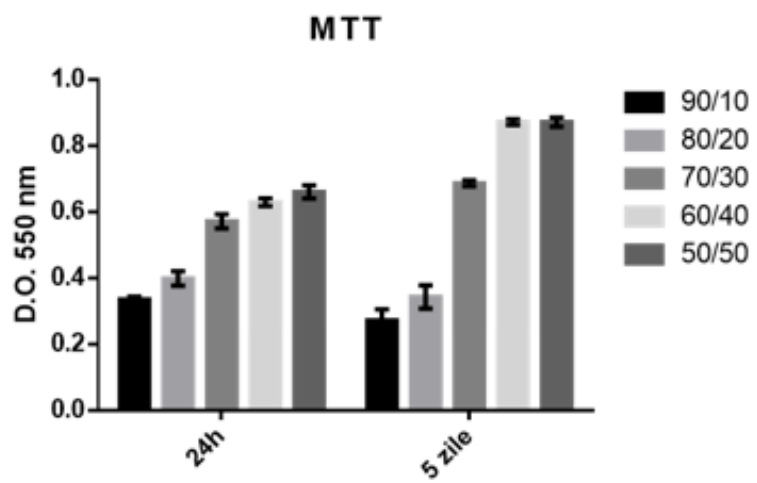

Fig. 6. Viability of 3T3-E1 cells cultured on PAA-MMNPs hydrogels after $24 \mathrm{~h}$ and 5 days seeding

GraphPrism software was also used to graphically represent the LDH results (figure 7). Magnetic nanocomposites do not exert cytotoxic effects on the cells after $24 \mathrm{~h}$. However, after 5 days, it is shown that the LDH activity profile in the culture medium depends on the hydrogels' composition. The PAA-MMNPs: 90-10 and 80-20 samples determines 
the highest increase of the LHD activity in the culture medium, so they exert a significant cytotoxic effect as compared to PAA-MMNPs 60-40 and 50-50.

LDH

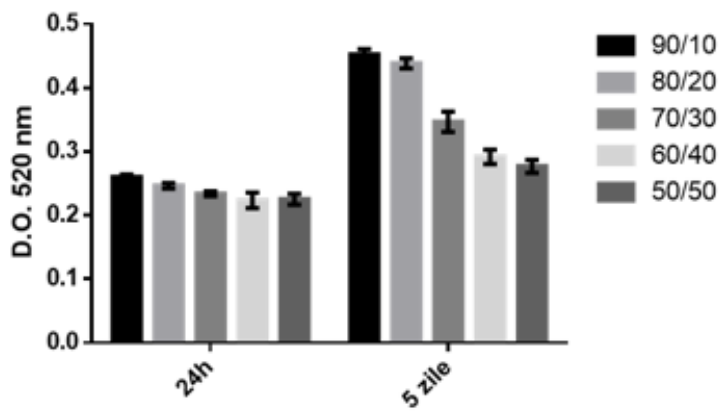

Fig. 7. Cytotoxic potential of PAA-MMNPs against 3T3-E1 cells after $24 \mathrm{~h}$ and 5 days seeding

In conclusion, 3T3-E1 cell viability seeded on the hydrogels based on PAA and functionalized magnetite varies with composition. After 5 days seeding time, the viability of PAA-MMNPs: 60-40 and 50-50 significantly increased as a proof of their proliferation. The hydrogels do not induce the increase of LDH activity in the culture medium immediately after seeding, but the enzyme activity profile changes in time. We may suppose that PAA-MMNPs: 90-10 and 80-20 hydrogels do not sustain cells attachment instead of showing cytotoxic effects.

\section{Conclusions}

This study reports the complex morphostructural characterization by SEM and TEM analysis of the nanocomposite hydrogels based on polyacrylamide and double bond modified magnetic nanoparticles. In vitro biocompatibility tests show good viability and good proliferative potential of the nanocomposites (especially for PAA-MMNPs: 60-40 and 50-50 composition). These nanocomposite hydrogels have great potential in the soft tissue engineering field as repairing scaffolds. Further studies will focus in vivo assays to sustain the in vitro biocompatibility results.

\section{References}

1.TARTAJ, P., MORALES, M.D.D., VEINTEMILlAS-VERDAGUER, S., GONZALEZ-CARRENO, T, SERNA, C.J., J. Phys. D: Appl. Phys., 36, 2003, p. R182-R197.

2.FARAJI, M., YAMINI, Y., REZAEE, M., J. IranChem. Soc., 7, no. 1, 2010, p. 1-37.

3.NIEMEYER C.M.,Angew. Chem. Int. Ed., 40, no. 22, 2001, p. 4128-4158.

4. COJOCARU, F.D., BALAN, V., POPA, M.I., LOBIUC, A., ANTONIAC, A., ANTONIAC, I.V., VERESTIUC, L., Int. J. Biol. Macromol., 125, 2019, p. 612-620.

5. GUAZZO, R., GARDIN, C., BEllin, G., SBRICOLI, L., FERRONI, L., LUDOVICHETTI, F.S., PIATTELLI, A.,ANTONIAC, I., Nanomaterials, 8, no.5, 2018, 349.

6. MATES, I., ANTONIAC, I., LASLO, V., VICAS, S., BROCKS, M., FRITEA, L., MILEA, C., MOHAN, A., CAVALU, S., Sci. Bull. B Chem. Mater. Sci. UPB, 81, no.1, 2019, p.206-16.

7. MARINESCU, R., LAPTOIU, D., ANTONIAC, I., PETCU, C., Key Eng. Mater.,583, 2014, p. 145-149.

8. JILES, D., Introduction to Magnetism and Magnetic Materials, $1^{\text {st }}$ ed.,Springer-Science+Business Media, Chapman and Hall, London, 1991, p. $1-425$.

9.MORRISH, A.H., The Physical Principles of Magnetism, $1^{\text {st }}$ ed., Wiley-IEEE Press, New York: IEEE Press, 2001, p. 1-700.

10. PANKHURST, Q.A., THANH, N.T.K., JONES, S.K., DOBSON, J., J PHYS D APPL PHYS., 42, no. 22, 2009, art.no. 224001.

11.KANG, S., WANG, Y.W., XU. X., NAVARRO, E., TICHAUER, K.M., LIU, J.T.C., J. Biophotonics, 11, no. 4, 2018 , art.no. e201700246.

12. BANGS L.B., Pure Appl. Chem., 68, no. 10, 1996, p. 1873-1879.

13.ZACARY, R.S., FORREST, M.K., MIQIN, Z., Mater. Today, 14, no.7-8, 2011, p. 330-338.

14.JIRI, K., YAZAN, H., LUKAS, R., ZBYNEK, H., MIRKO, C., VOJTECH, A., ONDREJ, Z., Nanomaterials, 7, no. 9, 2017 , art.no.243.

15. THOREK, D.L., CHEN, A., CZUPRYNA, J., TSOURKAS, A., Ann. Biomed. Eng.,13, no. 1, 2006,p.23-38.

16. LOPEZ-QUINTELA, M.A., TOJO, C., BLANCO, M.C., RIO, L.G., LEIS, J.R.,Curr. Opin. Colloid Interface Sci., 9, no.3-4,2004, p. $264-278$.

17. MOHAMMED, L., GOMAA, H.G., RAGAB, D., ZHU, J., Particuology, 30, 2017, p. 1-14.

18. GUPTA, A.K., GUPTA, M., Biomaterials, 26, no. 18, 2005, p. 3995-4021.

19. JIN, R., LIN, B., LI, D., AI, H., Curr. Opin. Pharmacol., 18, 2014, p. 18-27.

20. TANASA, E., ZAHARIA, C., RADU, I.C., SURDU, A.V., VASILE, B.S., DAMIAN, C.M., ANDRONESCU, E., Nanomaterials, 9, no.10, 2019, art.no.1384.

Manuscript received: 6.11 .2019 